\title{
REVIEW OF HEAT TRANSFER AUGMENTATION FOR COOLING OF TURBINE BLADE TIP BY GEOMETRICAL MODIFICATIONS TO THE SURFACES OF BLADE
}

\author{
Sushil Sunil Gaikwad ${ }^{1}$, C. R. Sonawane ${ }^{2}$ \\ ${ }^{1}$ PG Student, Mechanical Engg Dept, Pimpri Chinchwad College of Engg, Pune, Maharashtra, India \\ ${ }^{2}$ Asso. Prof, Mechanical Engg Dept, Pimpri Chinchwad College of Engg, Pune, Maharashtra, India
}

\begin{abstract}
In today's industrial scenario, Gas Turbine is one of the most important components of auxiliary power plant system. In order maximize the overall performance and efficiency of all modern turbines, which theoretically operate according to Brayton cycle, they are operated at a very high temperature. These temperatures are so high that, which may fall in the region of turbine blade material melting point temperatures. Due to such high temperatures there is a possibility that the turbine blades may get damaged due to produced thermal stresses and presents a possible threat to the turbine system as well as the operators. Hence to ensure safe and reliable working of the turbines an effective and reliable cooling system is necessary.Currently available methods for cooling of the turbine blades include film cooling with impingement cooling for the leading edge, rib turbulated cooling using serpentine passages for the middle portion of the blade and pin fin cooling for the trailing edge of the turbine blades. The cooling mechanism for turbine blades must include cooling for all possible regions which are exposed to hot gas flow. The turbine blade tip is one of the critical regions which are severely exposed to hot gas flow occurring due to the leakage of gases from the clearance gap between the turbine tip and the shroud. Hence the tip of the turbine blade must be cooled effectively to prevent thermal expansion of the turbine blade tip due to heating. This cooling will eventually help to avoid rubbing of blades to the shroud which may cause their wear. In this paper, we will be presenting the review of various efforts made by various authors towards the cooling of the turbine blade tip. The paper includes both, experimental methods developed as well as numerical efforts reported. Various experimental setups developed for turbine blade tip cooling includes the pioneer work of R. S. Bunker [1] to the recent efforts put by the Potdar et al. It has been noted that most of the authors had attempted this kind of problems experimentally only. They have found that the heat transfer can be improved by adding various types of protrusions on the flat plate surface. These added surface essentially help to produce vortex kind of structure and eventually increases the turbulence level near the tip surface. However it is also fact that to carry out the experimentations for various conditions is very costly due to the need of the today's sophisticated measuring devices required to understand and visualize the heat transfer phenomena. On the other hand numerical simulations will provide the detailed visualization and analysis of the heat transfer and flow characteristics for cooling of turbine blade tip. However producing the accurate and reliable results using available CFD software ANSYSFluent also need to be reviewedAn attempt here is to explore and present most of the recentcontributions presented by various authors. These reviews will help and provide the detailed guidelines for planned numerical and experimental investigations required for the cooling of turbine blade tip, which help to provide the feasible and practically usable solution for cooling turbine tip.
\end{abstract}

Keywords - Turbine Tip Cooling, Turbulence, numerical simulation, hotspots. ****

\section{INTRODUCTION}

Modern gas turbines operate at very high temperatures for increasing the efficiency and performance of the turbines. But these high temperatures may exceed the material melting temperature of the turbine blades. Hence proper cooling system must be employed for the cooling of the turbine blades for their long life.

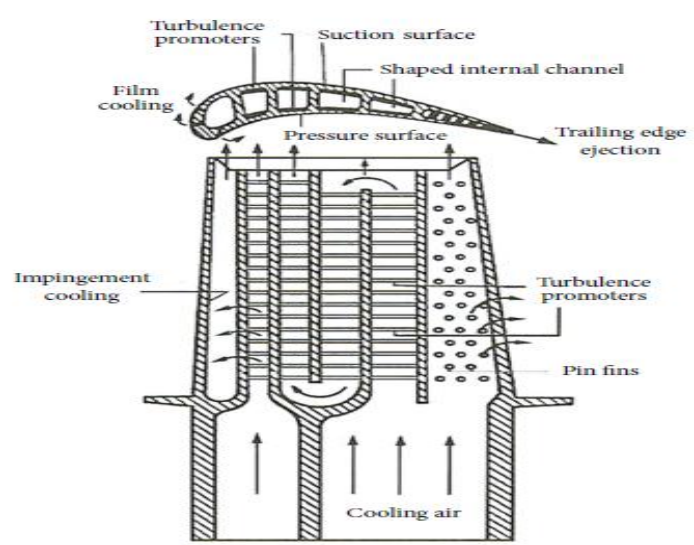

Fig1. Typical cooling techniques for turbine blades 
At present many cooling techniques for the internal and external cooling of the turbine blades are in practice. Fig 1 shows typical cooling techniques for the cooling of internal and external regions of the turbines. The leading edge of the turbine blade airfoil is cooled by using impingement cooling with film cooling, the middle portion is cooled by using rib turbulated cooling and the trailing edge is cooled by using pin fin cooling with ejection. The cooling of the turbine blades must include all the regions exposed to hot gases. One of such regions of a blade in case of high pressure turbines is the tip of the blade. For the mechanical and thermal expansion of the blades a clearance gap is provided between the turbine blade tip and the shroud/ fixed casing of the turbine. But due to this clearance gap, leakage of hot gases occurs mainly due to the difference between the pressure side and suction side of the blade.

And this gap cannot be completely eliminated since it will cause rubbing of the blades to the casing thereby causing damage to the turbine. Hence the cooling of the turbine blade tip must be given a special attention for safe and efficient working of the turbines. One very common method of cooling is by providing serpentine passages in the middle portion of the turbine blade. Fig 2 shows the serpentine passages commonly used for the internal cooling of turbine blades.

There are several publications available discussing the effects of providing ribs/vanes/pin fins inside these serpentine passages which improve the heat transfer by considerable amount.

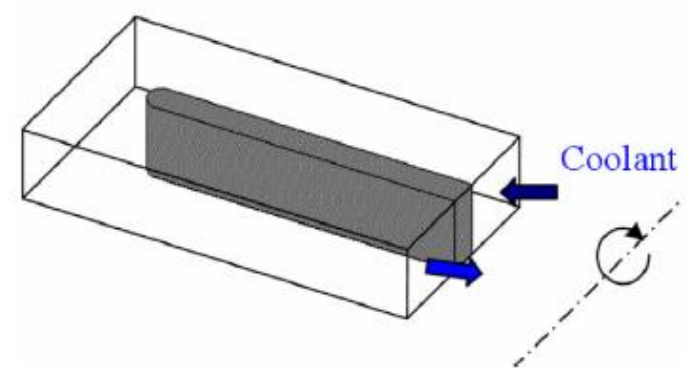

Fig2. A two pass serpentine passage inside a turbine blade

A slight modification in the method of tip cooling involves direct geometrical modifications to the internal tip of the turbine blades. Ron S. Bunker [1] experimentally investigated the effect of providing pin arrays to the internal tip cap of turbine blades. Five different configurations were used to compare the heat transfer coefficients. A smooth surface, two different heights of aluminium pin arrays, one more closely spaced array and one pin array made of insulating material were experimentally tested. As compared to smooth tip it was found that the provision of pin arrays showed heat transfer coefficient improved by a factor of 2.1. Also this method resulted in negligible pressure drop as compared to that of a smooth surface of the turbine internal tip. Gongnan Xie and Bengt Sunden [2] simulated the same geometry as given by Bunker. Only here the difference was provision of hemispherical dimples/concave dimples on the internal tip cap instead of arrays in case of Bunker's experiment. Here the flow was numerically simulated and studied by using two different heights of dimples were used. It was found that the heat transfer was doubled compared to smooth tip by provision of dimples on the surface of internal tip cap of the turbine blades. In yet another analysis Gongnan Xie [3] carried out numerical simulations for six different arrangements of the guide rib/vanes in a two pass serpentine passage. The guide ribs/vanes were placed at different locations inside the passage and their effect on the heat transfer coefficient was examined numerically using computational fluid dynamics technique. It was concluded that the tip heat transfer coefficients of channels with guide ribs/vanes are upto $65 \%$ higher than those of a channel without the provision of guide ribs/vanes. Another conclusion made was that there would be improved heat transfer if the guide ribs/vanes are properly placed inside the channel. But this method was found o be less effective as compared to the direct geometrical modifications as proposed by Bunker. Another option of tip cooling was proposed by Gongnan Xie and Bengt Sunden [4] which stated the use of pin fins for the cooling of tip of the turbine blades. Also noteworthy was the point that the heat transfer enhancement for the pin fins is higher than that for the pin arrays once these pin fins are properly placed on the tip of the blade.

A very limited data is available and few researches have been conducted on the cooling of turbine blades by direct geometrical modifications to the internal tip cap. All the publications explaining this method have considered smooth channels without ribs i.e. assuming that the channels have constant heat transfer surfaces. Hence the heat transfer enhancement for turbine tip has been studied by considering smooth channels instead of ribbed ones.

In this paper, we will be presenting the review of various efforts made by various authors towards the cooling of the turbine blade tip. The paper includes both, experimental methods developed as well as numerical efforts reported. Various experimental setups developed for turbine blade tip cooling includes the pioneer work of R. S. Bunker [1] to the recent efforts put by the Potdar et al. It has been noted that most of the authors had attempted this kind of problems experimentally only. They have found that the heat transfer can be improved by adding various types of protrusions on the flat plate surface. These added surfaces essentially help to produce vortex kind of structure and eventually increases the turbulence level near the tip surface. However it is also fact that to carry out the experimentations for various conditions is very costly due to the need of the today's sophisticated measuring devices required to understand and visualize the heat transfer phenomena. On the other hand numerical simulations will provide the detailed visualization and analysis of the heat transfer and flow characteristics for cooling of turbine blade tip. However producing the accurate and reliable results using available CFD software ANSYS-Fluent also needs to be reviewed. An attempt here is to explore and present most of the contributions presented by various authors. These reviews will help and provide the detailed guidelines for planned numerical and experimental investigations required for the cooling of turbine blade tip, which help to provide the feasible and practically usable solution for cooling turbine tip. 


\section{REVIEWS}

Ron S Bunker [1] demonstrated a method to increase the convective heat flux on the internal cooled tip cap of turbine blade. The method involved many variations to the internal tip cap including placemen of arrays of discrete shaped pins. As the flow inside the passages takes a turn by $180^{\circ}$, the augmentation and mechanism had been designed to contain impingement like flow, channel flow and strong secondary flow. A large scale model of a sharp $180^{\circ}$ turn was used along with liquid crystal thermography method to obtain the heat transfer distributions over the tip surface. The inlet Reynolds number ranged from $2,00,000$ to $4,50,000$ in his study. The inlet and exit passages had aspect ratios of $2: 1$, while the tip turn divider to-cap distance maintains nearly the same hydraulic diameter as the passages. The details of the geometry are as shown in the fig. 3 below. The overall test model is composed of a two-pass serpentine with sharp 180 deg tip turn as depicted in Fig. 3., where the shorter walls are the airfoil pressure and suction sides. The two-pass tip turn model was scaled up to achieve a range of test Reynolds numbers from 200,000 to 450,000 with atmospheric pressure discharge while maintaining a large enough size for full-surface data, as well as a small enough flow rate for in-house air supply. Each channel is $13.97 \mathrm{~cm}$ by $6.99 \mathrm{~cm}$ in size, with hydraulic diameter of 9.32 $\mathrm{cm}$. Corresponding channel Mach numbers are from 0.091 to 0.192 . A bellmouth inlet was used for the test model to reduce any inlet distortions. The inlet channel length is about ten hydraulic diameters. Both inlet and exit channels are smooth, without turbulators Only the tip internal surface is heated in this model, which ignores any effect due to an upstream thermal flow development. The Figure 3(a) shows the assembly view of the test model. The internal separation rib is $2.54 \mathrm{~cm}$ thick, making the full tip-cap section $3.97 \mathrm{~cm}$ by $16.51 \mathrm{~cm}$. A distance of $8.89 \mathrm{~cm}$ was used for the flow gap between the rib end and the tip cap (fig 3(b)), about the same as the channel hydraulic diameter as a rule of thumb. The tip and other walls are made of $2.54 \mathrm{~cm}$ thick acrylic. Each test surface is formed of a $0.762 \mathrm{~mm}$ thick base aluminum plate with the desired features machined integrally. The liquid crystals and heater are trapped between the acrylic and the test surface. The color response of the liquid crystals is viewed from the outside of the model tip turn surface. Surface temperatures are measured utilizing the liquid crystal video thermography method. A wide band liquid crystal Hall crest 40C5W pre applied to a Mylar sheet was calibrated over its entire color band. A curve fit of liquid crystal hue verse calibration temperature was then used to calculate wall temperatures. The wall heater system was a stack up consisting of $2.54 \mathrm{~cm}$ of acrylic insulation, liquid crystal sheet, adhesive, foil heater, adhesive, and a $0.762 \mathrm{~mm}$ nominal aluminum plate. A thin aluminum plate was used to allow for machining of thermal enhancement features while minimizing thermal resistance. Though very thin, this plate will still result in some thermal spreading of the local heat transfer data; the main effects and even many details will still be apparent. A uniform heat flux boundary condition is created by applying highcurrent, low-voltage dc power to the foil heater. Liquid crystal images were taken with an red-green-blue RGB charge coupled device CCD camera.

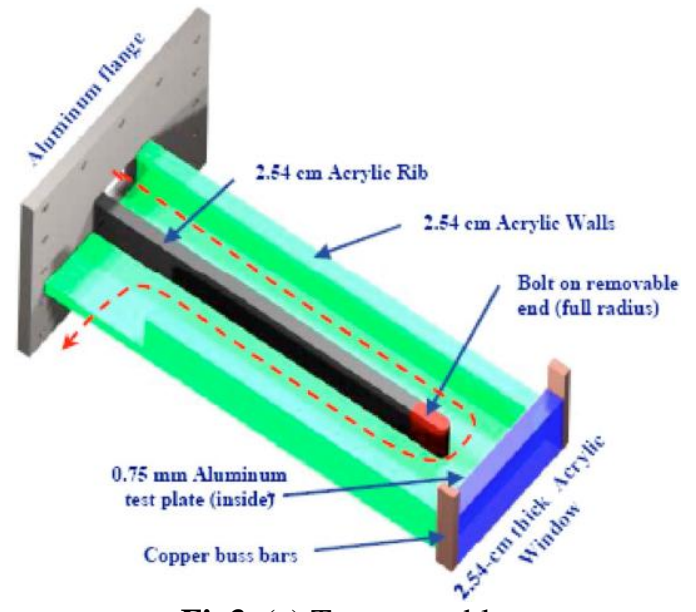

Fig3. (a) Test assembly

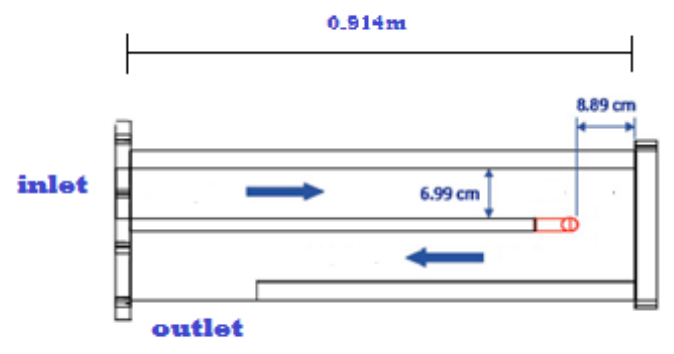

Fig3. (b) The geometry under consideration

Each data set is comprised of four to six images taken at different heat flux settings. Heat losses were measured to be less than $2 \%$ of the total power input, as determined by nonflowing and internally insulated powered tests over the range of heater settings and also monitored by outside surface thermocouples. Radiation heat transfer is negligible in this test section relative to the total power and convective heat transfer. The insulating material of the pins has a very low thermal conductivity of $1 \mathrm{~W} / \mathrm{m} \mathrm{K}$. Five tip-cap surfaces were tested along with a smooth surface, two different heights of aluminum pin arrays, one more closely spaced pin array, and one pin array made of insulating material. The heat transfer coefficients as compared to the smooth surface area were increased by up to a factor of 2.5. Most of this increase is due to the added surface area of the pin array. However, factoring this surface area effect out shows that the heat transfer coefficient has also been increased by about 20-30\%, primarily over the base region of the tip cap itself. This augmentation method resulted in negligible increase in tip turn pressure drop over that of a smooth surface which is a noteworthy feature of Bunker's experimentation. As seen from fig 4 the various surfaces and their avg Nusselt number were experimentally investigated for different Reynolds numbers. The standout heat transfer performance in this comparison is that of the tall pin array. This surface treatment provides both increased active surface area and augmentation of the heat transfer coefficients through flow turbulation and disruption. 


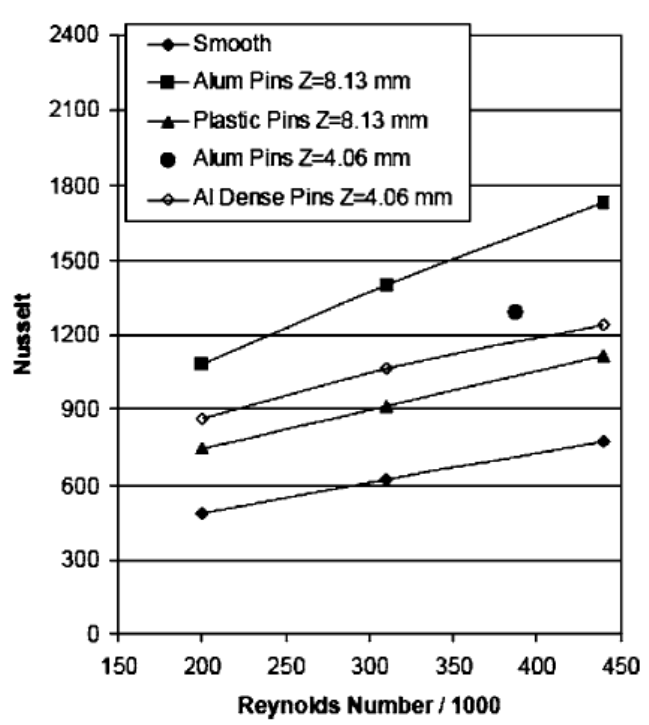

Fig4. Surface average Nu for all test surfaces

The best heat transfer performance in this comparison was given by the tall pin array. This surface treatment provides both increased active surface area and augmentation of the heat transfer coefficients through flow turbulation and disruption. The benefits of this configuration also increase with $\mathrm{Re}$ to a greater degree, overcoming the tendency seen in the other results to lose enhancement magnitude. The surprising comparison is that the insulating pins performed nearly as well as the dense/shorter pin array. This is due to the benefits of flow turbulation when spacing allows such interactions over a larger surface area that has restricted flow access to the pins. The comparison shown in Fig. 4 is on the basis of what the engine component will experience, namely, the combined effect of increased surface area and augmented heat transfer coefficients. The importance of the pin array's ability to turbulate the flow over the primary surface is emphasized here. It is also seen that this portion of the overall effect is substantially diminished as the $\mathrm{Re}$ number is increased. The new augmentation technique also resulted in a negligible pressure loss which proved to be of a great importance.

Another experimental attempt was made by Potdar et al [5] for the internal cooling of turbine blades. Two types of ribs were used; one $45^{\circ}$ arc of circle and another $60^{\circ}$ arc of circle rib. The configuration of the ribs is as shown in the figure 5 . The shape of the ribs was the same for both the configurations used only the difference being of that of the angle of inclination of the ribs with respect to the passage. The internal passages were provided with ribs on the opposite walls. A stationary square channel with aspect ratio of 1 was set up for the analysis. The hydraulic and thermal performance and characteristic was measured with the help of Nusselt number and frictional factor. Square ribs $(\mathrm{w} / \mathrm{e}=1)$ were considered as the baseline configuration.

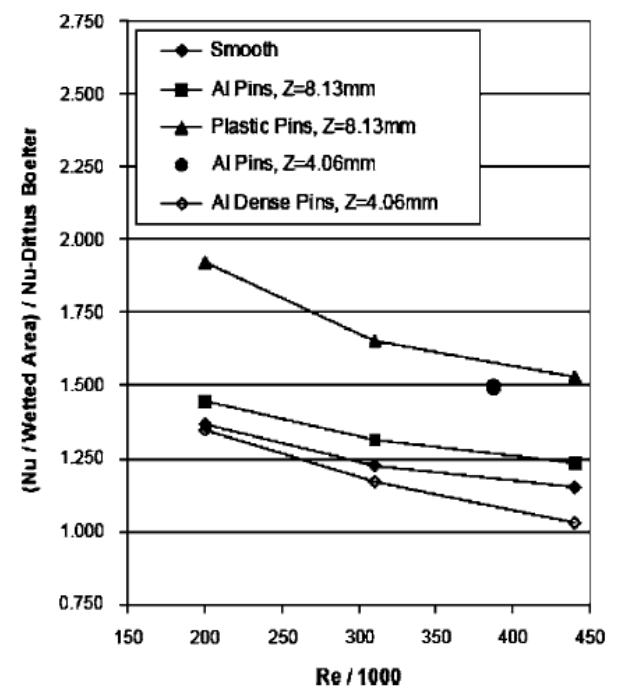

Fig4. Effect of pin array turbulation ability on heat transfer augmentation

Rib geometries, comprising three rib height-to-channel hydraulic diameter ratio (blockage ratio) of $0.083,0.125 \&$ $\mathbf{0 . 1 6 7}$ as well as rib spacing (pitch to height ratio) was $\mathbf{1 0}$.

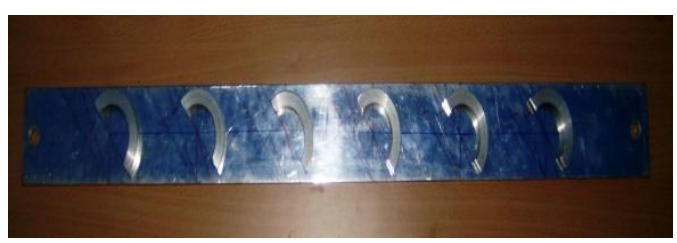

Fig5. Angled arc of ribs

The heat transfer performance for the channel was calculated for Reynolds numbers 40,000 to 75,000. It was concluded from the experiments that the increase in width of the ribs increased the thermal performance of the channels increases. By the combined effect of rib width, rib spacing and flow parameters, the optimal cooling configuration was obtained for the stationary square channel.

Gongnan Xie and Bengt Sunden [2] investigated numerically the heat transfer augmentation for the same geometry as that of Bunker. The difference being the provision of hemispherical dimples instead of pin arrays as considered by Bunker. The arrays of dimples were provided on the internal tip cap of the turbine blade as shown in fig 6 . The turbulent convective heat transfer between the fluid and dimples and heat conduction within the dimples and the tip were computed at the same time. The inlet Reynolds number range was from 100000 to 600000. As already defined by Bunker the geometry gave rise to impingement like flow and due to the provision of dimples it gave rise to dimple-induced advection flow in this case. It was found that the heat transfer coefficient of the dimpled tip was upto two times higher than the smooth tip with less than 5\% pressure drop which is acceptable for the reliable operation of the turbines. 


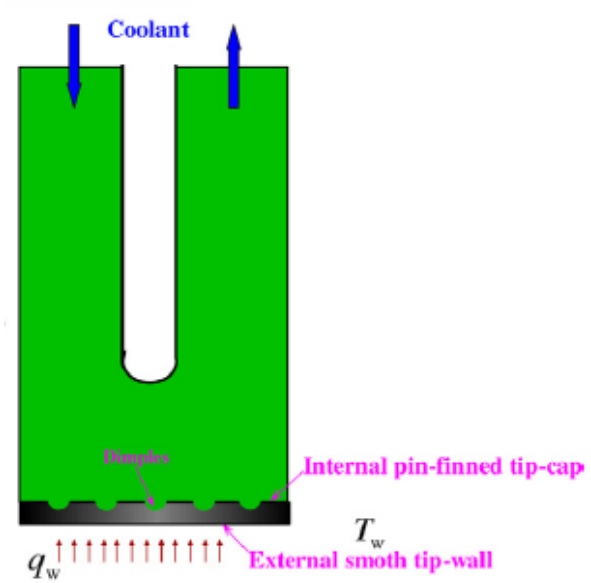

Fig6. Dimples on the internal tip cap

A realizable $\mathrm{k}-\varepsilon$ turbulence model was used for computations based on the previous researches as it gives one of the best agreements between the calculated and experimental data that is available. Two different heights of the pins were used for comparison - one short and another tall. The calculated results were validated with the experimental data and it was concluded that the provision of dimples on the internal tip cap provided twice the heat transfer as compared to that of a smooth channel along with a low value of pressure drop. If the height of the dimples is decreased it leads to a slightly lower heat transfer at high Reynolds number. This may be due to the low surface area available for interaction with the dimples. A comparison of the obtained results is as shown in fig.7. From fig.7 (a) we see that the overall Nusselt number for a dimpled tip channels are higher than those for a smooth tip both for Case I and Case II. The highest factor of heat transfer enhancement for the two cases is a factor of 2 and lowest is around 1.35. It is observed that the Case I offers slightly higher heat transfer enhancement at high Reynolds number. But in case of pressure drop the smooth channels offer $2-5 \%$ less pressure drop as compared to the dimpled tip channels. But if the augmentation of heat transfer is considered this pressure drop is very less. Another comparison made by the author was between the pumping powers to the three channels and the heat transfer coefficient as shown in the fig 8 .

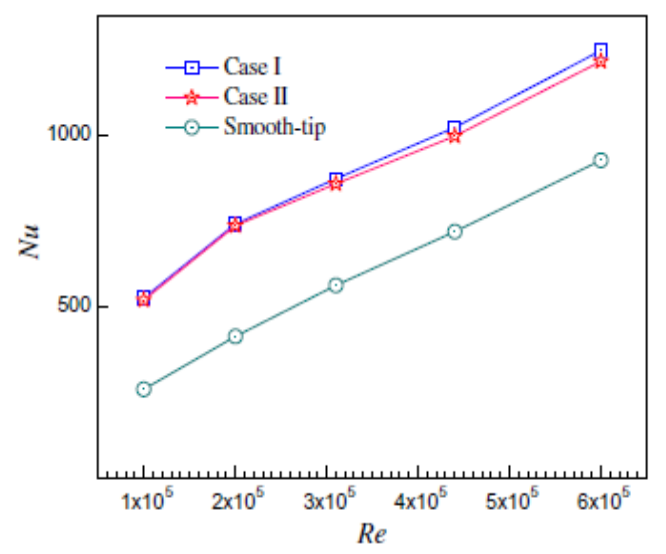

Fig 7(a) Heat Transfer

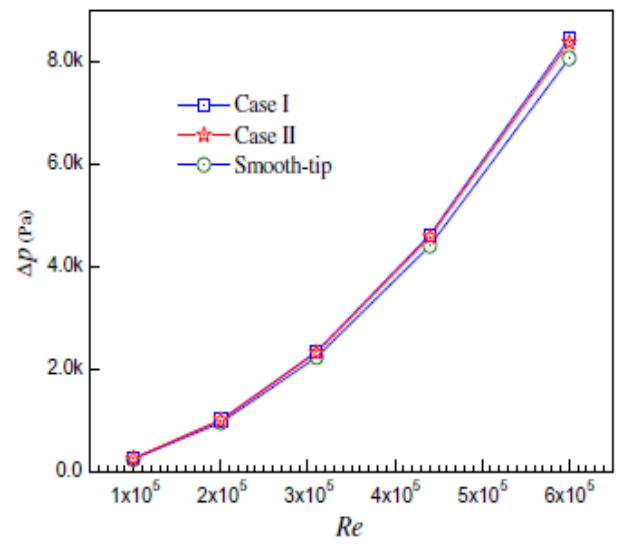

Fig 7(b) Pressure Drop

From fig. 8 we can see that for the same values of the power input the dimpled tip channels show an increased value of the heat transfer coefficient as compared to the smooth channel.

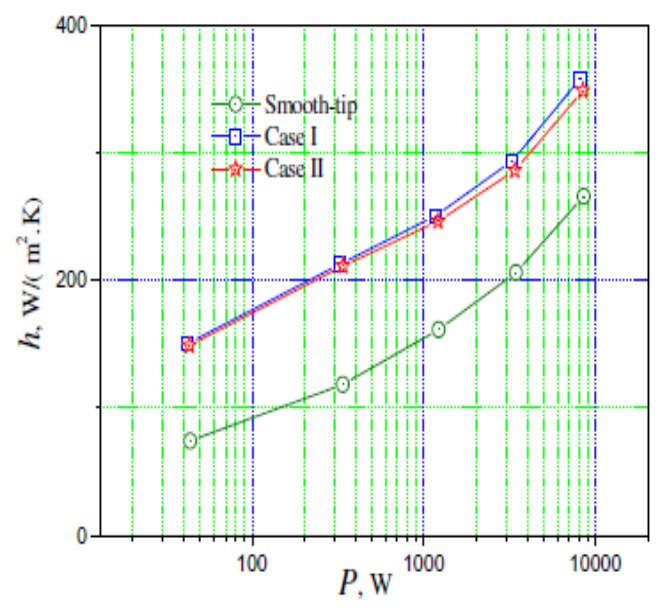

Fig 8 Performance comparison of here channels

Hence dimpled tips could prove a better option for turbine tip cooling with a small pressure drop that may be within the acceptable range of working of the turbine. From the above study it was found that dimples may prove to be a good option for the cooling of the turbine blade tips providing as much as twice the heat transfer as compared to that of a smooth channel. The use of ribs in the internal passages of the turbine blades, the provision of arrays of pins on the turbine blade tip and the provision of dimples to the internal tip cap of the turbine blades has shown a significant increase in the heat transfer of turbine blades as far as the computations and experimental results are considered. Yet another attempt was made by Gongnan Xie et al [3] to numerically simulate the serpentine passages of turbine blade to evaluate the heat transfer but this time considering the provision of pin fins to the internal tip cap of the turbine blades. The averaged heat transfer is similar to that determined by Bunker experimentally. The geometry used for simulation was same as that given by Bunker only the change being in the pin fin configurations and the experimentations carried out also differ. 


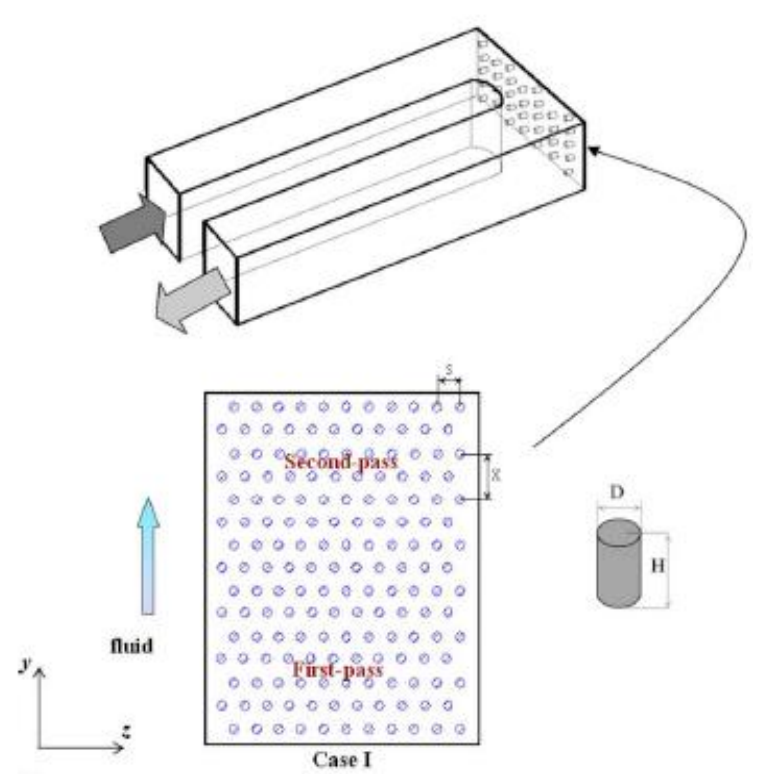

Fig9 Geometry for pin finned tip.

It was found that due to the provision of pin fins and due to the combination of turning, impingement and pin fin cross flow, the heat transfer of the tip with pin fins was 2.67 times higher than that for smooth tip but with a penalty of pressure drop of around $30 \%$. The geometry is as shown in the fig.9. A renormalization group $\mathrm{RNG} \mathrm{k}-\varepsilon$ turbulence model was considered in this case because the experimental data was better than the other turbulence models. The geometry used for the simulation was same as that given by Bunker [1] and also the concept of pin fins is similar to that given by Bunker. The arrangement of the pin fins was different in this case and also the analysis conducted was different.

Table1 (a) Cases considered

\begin{tabular}{|l|l|l|}
\hline Model & $\begin{array}{l}\text { Diameter } \\
\mathbf{D}(\mathbf{m m})\end{array}$ & $\begin{array}{l}\text { Streamwise Pitch } \\
\mathbf{X}(\mathbf{m m})\end{array}$ \\
\hline Case 1 & 4.064 & 21.117 \\
\hline Case 2 & 4.064 & 12.192 \\
\hline Case 3 & 4.064 & 12.192 \\
\hline Case 4 & 4.064 & 7.62 \\
\hline Case 5 & 4.064 & 7.62 \\
\hline Case 6 & 6.096 & 18.288 \\
\hline Case 7 & 3.048 & 12.192 \\
\hline Case 8 & 6.096 & 12.192 \\
\hline Case 9 & 3.048 & 12.192 \\
\hline
\end{tabular}

As seen from the fig. 9, the tip of the turbine blade was provided with pin fin structures. The pin fins were circular straight cylinders and were placed in a staggered arrangement. Nine models with different pin-fin parameters height $\mathrm{H}$ and diameter D and layouts pitches $\mathrm{X}$ and $\mathrm{S}$ are considered in this study. The details of the cases considered are as shown in the Table 1 (a) and (b).
Table 1(b) Cases considered

\begin{tabular}{|c|c|c|c|}
\hline Model & $\begin{array}{ll}\text { Spanwise } & \\
\text { Pitch } & \text { S } \\
(\mathrm{mm}) & \\
\end{array}$ & $\begin{array}{l}\text { Height } \\
\text { H (mm) }\end{array}$ & $\begin{array}{l}\text { Number } \\
\text { of pin } \\
\text { fins } \mathrm{N}\end{array}$ \\
\hline Case 1 & 6.096 & 8.128 & 165 \\
\hline Case 2 & 10.559 & 8.128 & 162 \\
\hline Case 3 & 10.559 & 4.064 & 162 \\
\hline Case 4 & 6.599 & 8.128 & 389 \\
\hline Case 5 & 6.599 & 4.064 & 389 \\
\hline Case 6 & 15.838 & 8.128 & 68 \\
\hline Case 7 & 10.559 & 8.128 & 162 \\
\hline Case 8 & 10.559 & 8.128 & 175 \\
\hline Case 9 & 10.559 & 4.064 & 162 \\
\hline
\end{tabular}

The comparisons for different cases are shown in the fig 10(a) and fig 10(b). Figure 10 provides the averaged Nusselt number and friction factor, as normalized by the corresponding values for the turbulent flow and heat transfer inside the smooth-tip channel, based on inlet channel conditions. From this figure, as expected with an increase in the Reynolds number, it is obvious that the value of $\mathrm{Nu} / \mathrm{Nu}_{0}$ is decreased while the value of $\mathrm{f} / \mathrm{f}_{\mathrm{o}}$ is increased. Case 4 and Case 5 provide the lowest heat transfer enhancement, while Case 7 provides the largest heat transfer enhancement below the Reynolds number of about 450,000 . The results indicate that increasing the number of pin fins does not mean an increased heat transfer enhancement.

By summarizing Fig. 10, the conclusion can be drawn that the heat transfer and pressure drop of the pin-finned tip two-pass channel are higher than those of the smooth tip two-pass channel. The benefit of heat transfer enhancement is substantial, even though the additional pressure loss is taken into account. Adopting pin fins increases the tip heat transfer, and hence, improves the cooling of the blade tip being exposed to high thermal load. A comparison of Nusselt numbers between this work and the experimental data by Bunker [1] is plotted in Fig. 11. Three cases, i.e., Case 1, Case 2, and Case 7 are used for examples. The reason for this selection is that the pin fins of Case 1 and Case 2 have the same geometry as those in the experiments, and for the experimental data, the case with the highest Nusselt number is chosen. It is found that good agreement of Nusselt numbers between of Case 1 and that case in Ref [1] is achieved. At high Reynolds numbers, Nusselt numbers of that case in Ref [1] are about 4-12\% higher than those of the present: Case 2 and Case 7. This might partly be due to the fact that in work by Bunker [1] shaped pins having different base fillet and tip radius were tested while in the present paper, constant cross section straight circular pins have been assumed for simplicity. Since the casting process does not make a perfect cylinder pin, a fillet needs to be placed at the endwall. The pins tested by Bunker [1] seem to be of a stepped-diameter type, having the largest diameter at the base, and then a gradually reduced diameter along the pin height until the pin tip. With the different layout of the various shaped pin fins, the augmented area factors are different. Another reason might be the heat flux boundary condition. 

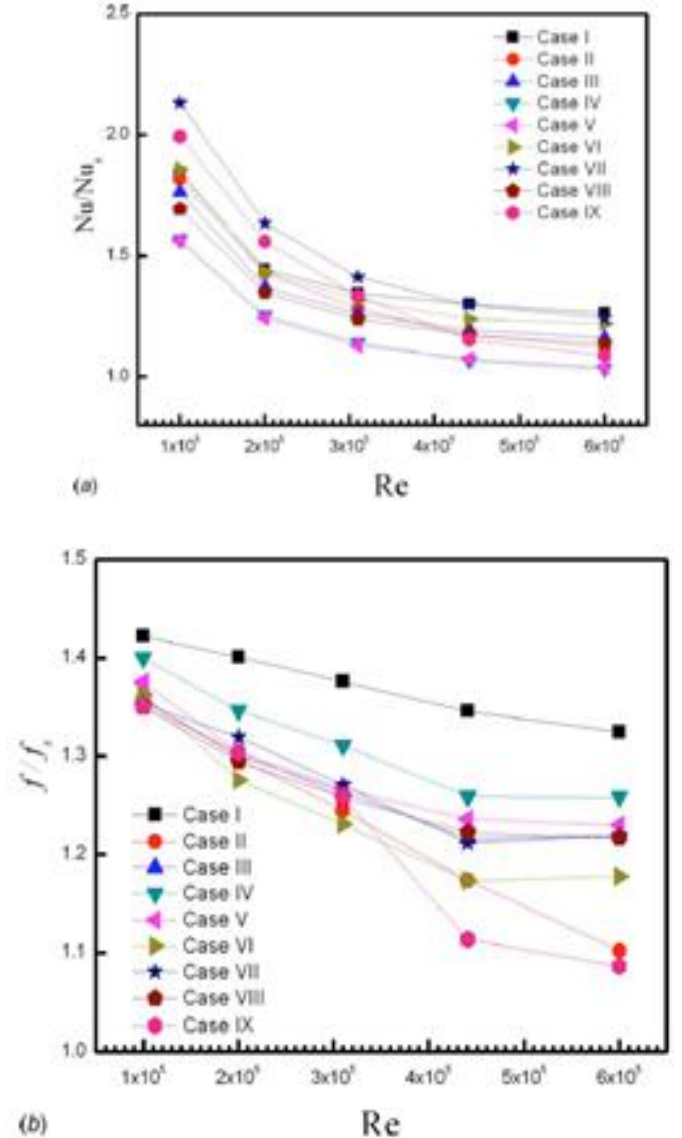

Fig 10 Normalized Nusselt number and friction factor comparedto smooth tip

In the experiments by Bunker [1], a uniform heat flux was created for the external tip cap by applying a heater under the endwall surface, while for simplicity in the present numerical study, and as the details of the profiled pin surface heat flux could not be acquired, a uniform heat flux boundary condition is prescribed on both the pin-fin surfaces and the endwall surface. The imperfection of the heat flux boundary condition may therefore contribute to the difference between the simulated results and the experimental data, since in practice; the pin-fin surfaces do not have a uniform heat flux. These reasons probably explain why the enhanced heat transfer of the present pin-finned tip is not similar as those of Bunker's pinfinned tips [1].

From the above experiments the conclusions made were as follows. The pin fins are very effective heat transfer enhancement devices for gas turbine blade tips due to combination of turn, impingement, and pin-fin. The pin fins force the vortices toward the tip wall, and thereby improve the turbulent mixing of the approaching cold and hot fluids near the tip. Compared with the smooth-tip channel, the heat transfer enhancement of the pin-finned tip channels is up to 2.67. The pin-fin heat transfer is about $30-40 \%$ higher than the endwall heat transfer. For the short small pin fins studied in the present paper, the heat transfer enhancement is influenced slightly by the pin fin height, but is affected clearly by the pinfin. The performance of heat transfer enhancement on the pinfinned tip depends on the pin-fin configurations and arrangements. By applying two criteria, it is found that the pinfinned tip channels provide good overall performance. It is suggested that application of pin fins for tip cooling is effective with a high heat transfer enhancement at a moderate additional pressure loss.

\section{CONCLUSIONS}

From the above discussed reviews and details one thing can be concluded that if the turbine tip is provided with some kinds of protrusions, it will cause turbulence near the tip region which will ultimately produce vortex structures near the tip wall. These vortices will allow the heat transfer to increase near the tip surface thereby reducing the temperature of the tip which is the final goal. From the numerical and experimental data available to date about the turbine tip cooling we can conclude that pin fins can prove to be a great tool for the enhancement of heat transfer of the turbine blade tip if they are properly arranged and configured.
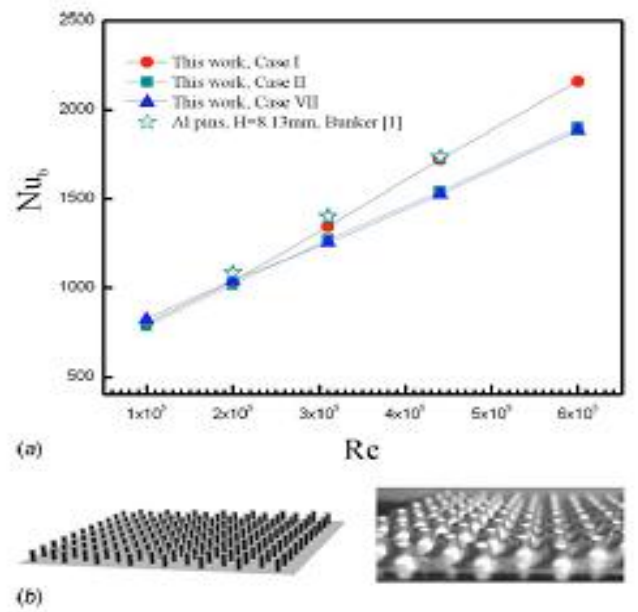

Fig11 Comparison between Simulated Results and Bunker's results [1]

Although the pin fins penalize with pressure loss, considering the increase in heat transfer as compared to smooth surface, this pressure loss is sufficiently low. The future scope of the current analysis includes the numerical simulation and analysis of different kinds of protrusions provided to the tip of turbine blade and their comparison with the experimental data available.

\section{REFERENCES}

[1] Ronald S. Bunker," The Augmentation of Internal Blade Tip-Cap Cooling by Arrays of Shaped Pins," Journal of Turbomachinery, OCTOBER 2008, Vol. 130 / 041007-1

[2] Gongnan Xie, Bengt Sundén.” Numerical predictions of augmented heat transfer of an internal blade tip-wall by hemispherical dimples," International Journal of Heat and Mass Transfer 53 (2010) 5639-5650.

[3] Gongnan Xie, Bengt Sundén, “Computational Analysis of Pin-Fin Arrays Effects on Internal Heat Transfer Enhancement of a Blade Tip Wall," Journal of Heat Transfer, MARCH 2010, Vol. 132 / 031901-1 
[4] Gongnan Xie, Bengt Sundén,Weihing Zhang, "Comparisons of Pins/Dimpls,Protrusions cooling concepts for a turbine blade tip wall at high Reynolds numbers," Journal of Heat Transfer, June 2011,Vol.133/061902-1

[5] Robert KIML, Sadanari MOCHIZUKI, Akira MURATA and Matej SULITKA, "Rib-Induced Secondary Flow Structures inside a High Aspect Ratio Trapezoidal Channel," Proceedings of the International Gas Turbine Congress 2003 Tokyo November 2-7, 2003.

[6] Je-Chin Han, "Recent Studies in Turbine Blade Cooling," International Journal of Rotating Machinery, 10(6): 443-457, 2004.

[7] Ravi Teja, S. Krishna Chaitanya, "Case study on Turbine Blade Internal Cooling," International Journal of Engineering Research \& Technology ISSN: 22780181, (IJERT) Vol. 2 Issue 3, March - 2013 ISSN: 2278-0181.

[8] Ana Paula P. dos Santos, Claudia R. Andrade and Edson L. Zaparoli, "Comparison of Different Gas Turbine Inlet Air Cooling Methods," World Academy of Science, Engineering and Technology 612012.

[9] S. Gupta, A. Chaube, Verma, "Review on Heat Transfer Augmentation Techniques: Application in Gas Turbine Blade Internal Cooling," Journal of Engineering Science and Technology Review 5 (1) (2012) 57-62. 